\title{
ExoSelect and ExoRequest: Targets and Resources for efficiently observing Exoplanets
}

\author{
Saeed Salimpour ${ }^{1,2,3,4^{*}, \text { Michael Fitzgerald }}{ }^{5,1}$, and Heath Demmert \\ ${ }^{1}$ Deakin University, Burwood, Victoria, Australia \\ ${ }^{2}$ International Astronomical Union, Office of Astronomy for Education, Heidelberg, Germany \\ ${ }^{3}$ Haus der Astronomie, Heidelberg, Germany \\ ${ }^{4}$ Max Planck Institute for Astronomy, Heidelberg, Germany \\ ${ }^{5}$ Las Cumbres Observatory, Goleta, CA, USA \\ "Corresponding author: astrophysics@saeedsalimpour.com
}

\begin{abstract}
In the past decade, exoplanet science has exploded, driven by discoveries using observations from both space-based and ground-based telescopes. Large amounts of data, coupled with technological advances and easy access to robotic telescopes, have allowed the general public and students to become vital contributors to the field. These developments have also provided fertile ground in the context of science education, by enabling exoplanet science to be taken into classrooms as an authentic scientific inquiry, echoing the notions of Science-as-Practice. This paper introduces technical infrastructure that enables beginners and students to quickly pick exoplanet targets and schedule an observation. It also provides a list of the "best" exoplanets to try and observe by month of the year (related to Right Ascension) and latitude (related to Declination). (무) $\odot \ominus 2021$ Astronomy Theory, Observations and Methods Journal
\end{abstract} Keywords: exoplanets - authentic data - educational resource - technology infrastrcuture https://doi.org/10.32374/atom.2020.2.6

\section{INTRODUCTION}

Over the past decade, there have been great strides in identifying and minimising the barriers that prevent students from engaging in authentic scientific inquiry using robotic telescopes in the classroom (Gomez \& Fitzgerald, 2017; Fitzgerald et al., 2014). One of the fundamental barriers is the time-pressure in schools, which is compounded by an overly busy curriculum (e.g.: Fitzgerald et al., 2019; Salimpour et al., 2020). In addition, the possibility of steep learning curves regarding technology and content knowledge can be discouraging for teachers who want to implement authentic scientific inquiry, especially those without appropriate support.

Various projects (e.g.: Brown et al., 2013; Fitzgerald et al., 2018; Reichart et al., 2005; Sadler et al., 2001) around the world have aimed at putting in place technical infrastructure that allows teachers to more easily overcome the above barriers, and focus on student learning in the context of both conceptual and epistemic practices of science (Lehrer \& Schauble, 2007). The aim is to develop flexible systems that can cater to everyone from the most basic to the most advanced, that enable students to focus on critical think- 


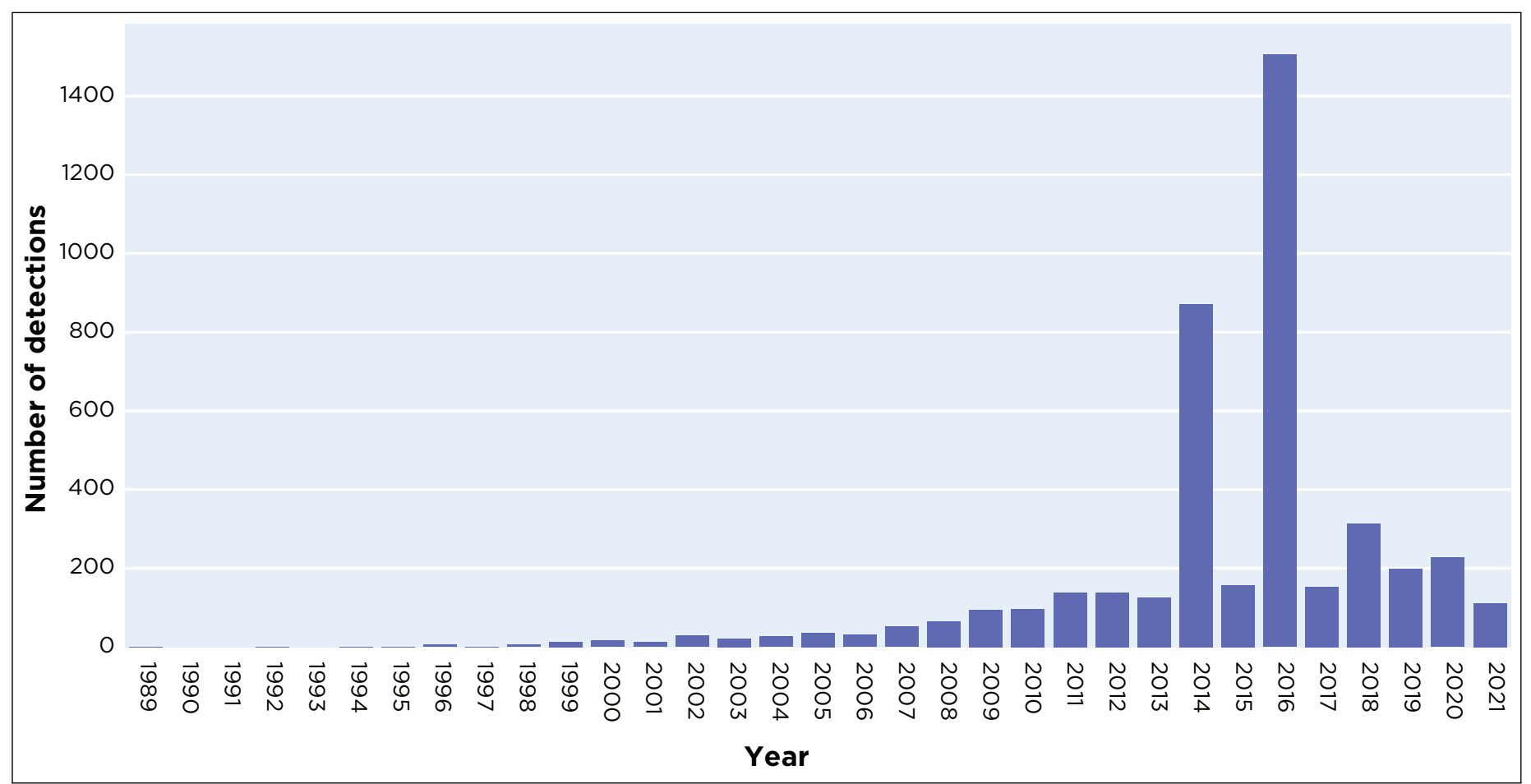

Fig. 1. Exoplanet detections over time, notice the two spikes (2014) and (2016), both associated with announcements of large-scale statistical analysis of data from previous years.

ing, essentially moving students from menial data 'reduction' to the inquisitive data 'analysis' (Fitzgerald, 2018).

This paper begins by providing a brief overview of exoplanet science, focussing on one of the most prolific and conceptually easier methods of detection. Then, the ways in which robotic telescopes have provided the opportunities for students to engage with and in authentic scientific inquiry are highlighted. Following this, the technical infrastructure that enables students and complete beginners to request observations of exoplanets is explained. The paper concludes by providing some perspectives on future directions for this endeavour.

\section{EXOPLANET SCIENCE}

In less than a decade, the number of exoplanet confirmations has exploded (see Figure 1), owing to the groundbreaking space-based observatory Kepler (Borucki et al., 2010; Howell, 2020). The current tally of confirmed exoplanets is 4455 (as of this writing), and the number of candidates yet to be confirmed is around 6297, so there is fertile ground to help astronomers confirm a vast number of exoplanets. Currently, the Transiting Exoplanet Survey Satellite (TESS) is continuously adding to this tally of exoplan- ets (Ricker et al., 2010; Guerrero et al., 2021).

The most prolific exoplanet detection method is the Transit Method (TM) (see Figure2), which is based on established and relatively simple laws of physics and geometry, some of which are taught at secondary school in curricula around the world (Salimpour et al., 2020). The key conceptual idea is grounded in the physics and mathematics of orbital motion, such as Kepler's Laws. With robotic telescope observations, the aim is to detect the dimming of the light from the host star, as an exoplanet or extrasolar planet "passes" in front of the star's disc. This geometry is represented in Figure 3. This is a simplified representational model, aimed at conveying the concept of detecting exoplanets via the Transit Method.

\section{ROBOTIC TELESCOPES}

The enabling technology that allows exoplanet science to be realised in the classroom is the use of Remote Robotic Telescopes (RRT). Gomez \& Fitzgerald (2017) provide an extensive overview of the role of RRT in education. The use of RRTs removes the technological, financial, and geographical barriers that are associated with allowing students access to researchgrade telescopes. Organisations like Las Cumbres Observatory (LCO) (Brown et al., 2013), Faulkes Tele- 


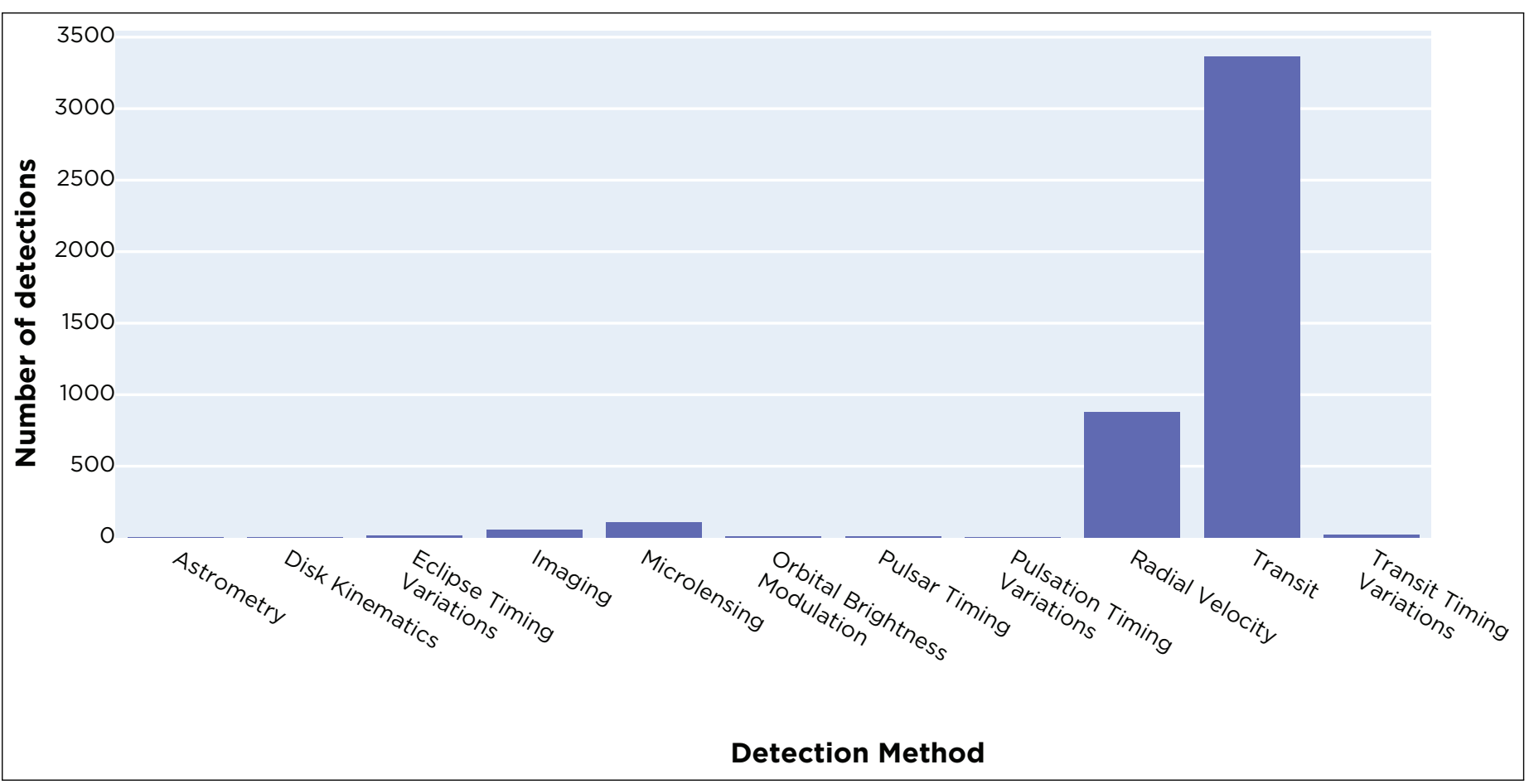

Fig. 2. Exoplanet detections methods, the Transit Method is by far the most prolific detection method, owing in part to the fundamental "simplicity" of measuring the dimming of starlight.

(a)

Simplified Orbital geometry (Perspective View)

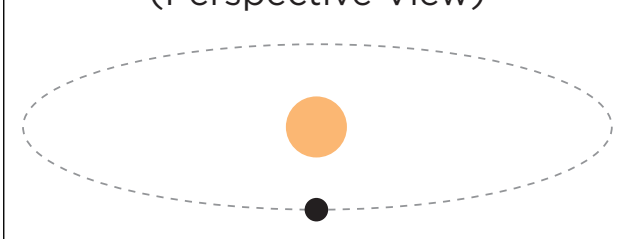

Simplified Orbital geometry (Side on View) (b)

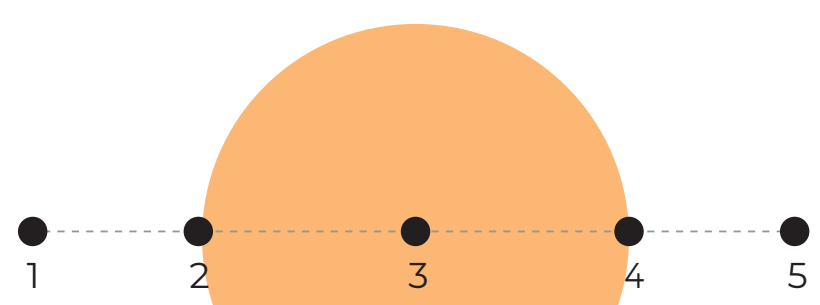

5

Time

Figures are not to scale

Fig. 3. Basic representation of the transit method, and (a) how the geometry of the orbit (b) allows for such a detection. The black dot represents the planet and the orange circle represents the host star. On the right, the planet is shown at five different positions relative to the star it is orbitting. Although this figure shows an ideal alignment where the planet is going directly through the middle of the star, in most cases the orbital alignment is not so ideally aligned. 


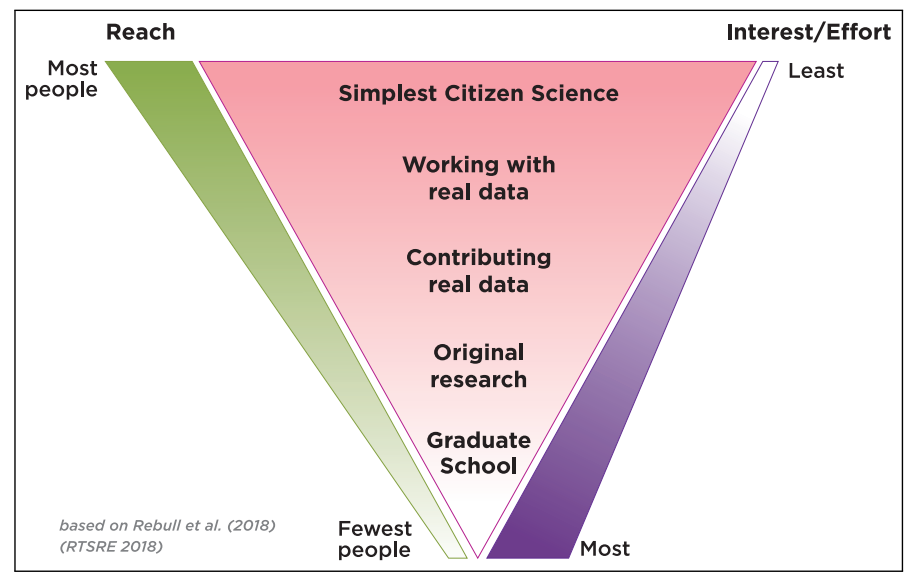

Fig. 4. Funnel of Interest ecosystem (modified from Rebull, 2018).

scope (Roche et al., 2008), SkyNet (Reichart et al., 2005), Microobservatory (Sadler et al., 2001) and SPIRIT (Luckas \& Gottschalk, 2018) amongst others, have enabled authentic scientific inquiry to be brought into the classroom. This has led, not only to richer experiences for both students and teachers, but has also allowed students to publish authentic research and collaborate with astronomers. This approach is quite different to typical Citizen Science projects in astronomy, where the data has been significantly cleaned up and presented in a more idyllic manner removing much of the potential noise in the data, rather, in this case students are appreciating the messy nature of authentic data (see Figure 5) that they have collected (Salimpour et al., 2021).

The interaction between the reach, in terms of breadth and size of the target population, and the effort required to undertake authentic investigations is conceptualised by Rebull (2018) via the Funnel of Interest (FoI) (see Figure 4). In essence, the investigations that use readily available data are more likely to reach a larger audience (which, in the case of Citizen Science, do not need to have significant prerequisite knowledge), require the least effort to engage with the science, and can attract those with little initial interest. Further down the FoI, the reach of the investigations decreases, as there is a move from already available data to new data which is collected by the student. Therefore, only those who are really interested will be engaged, as this requires more effort on the part of the student. In the context of a school classroom, more effort and scaffolding on the part of the teacher is required to guide the students through the known unknowns, and unknown unknowns.

\section{EXOPLANET SCIENCE IN THE CLASSROOM}

Realising exoplanet science in the classroom in a way that is true to the notions of authentic inquiry has many technical challenges that can be overwhelming to teachers and students. This is owing to the fact that teachers are under enormous time pressures, that most teachers lack the Pedagogical Content Knowledge (PCK) (Shulman, 1986) and practical scientific research skills (Fitzgerald et al., 2019), and that some of them are even teaching out of field (Luft et al., 2020). These issues prevent them from tapping into the enormous potential offered by this field of inquiry. Picking exoplanets, planning and requesting observations, analysing the data, and interpreting the findings each have their own challenges with their own requisite extended learning curves. However, these challenges can be overcome by careful educational design, which involves two key layers: Technical Infrastructure, and Education Resources (see Figure 6). The technical infrastructure, which is is the outer layer, enables students to quickly and efficiently request observations using robotic telescopes. The inner layer, which is the educational resources, is about providing teachers with a robust set of resources that they can be used to scaffold their students conceptually and technically in undertaking authentic scientific inquiry in the context of exoplanets in the classroom.

\section{TECHNICAL INFRASTRUCTURE}

\section{Exoplanet Targets}

One of the challenging aspects of making observations of exoplanets in the context of the classroom is determining which of the thousands of exoplanets would be a suitable observation target, taking into consideration the various pressures in schools (Fitzgerald et al., 2015, 2019). After several implementations of exoplanet science in high schools it became clear that for students to easily and fluidly engage with the science and analysis it would be best to have a robust list of provided targets. This is so students could quickly and efficiently pick a target simply relative to the month they were observing. This can provide relief from the excessive amount of time it takes to plan an observation.

Such a robust list, one that would allow students to conduct viable observations of known exoplanets, was complied using the NASA Exoplanet Archive - an extensive database of exoplanets from various missions (Fig. 7), a list of targets that would allow students to 


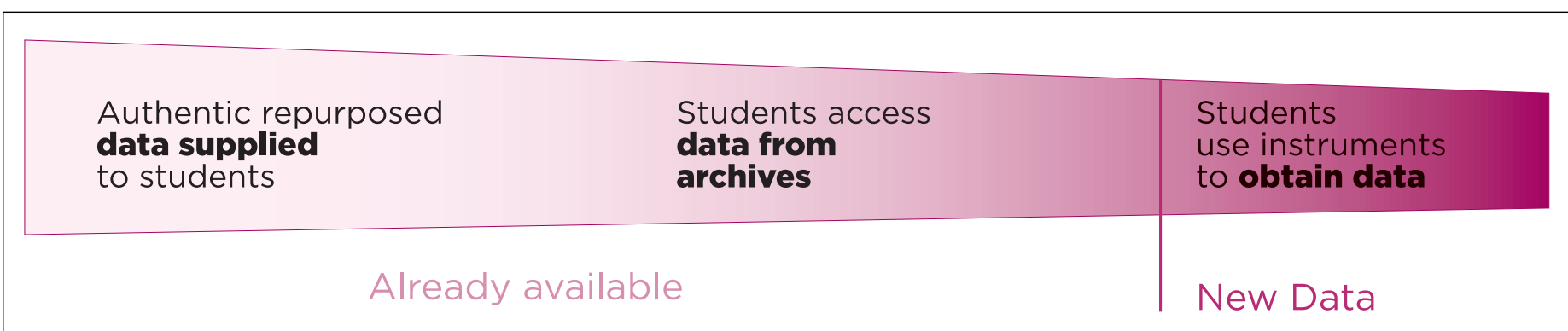

Fig. 5. The spectrum of Authentic Data. The use of Robotic Telescopes is situated at the far end of the spectrum - New Data (Salimpour et al., 2021)

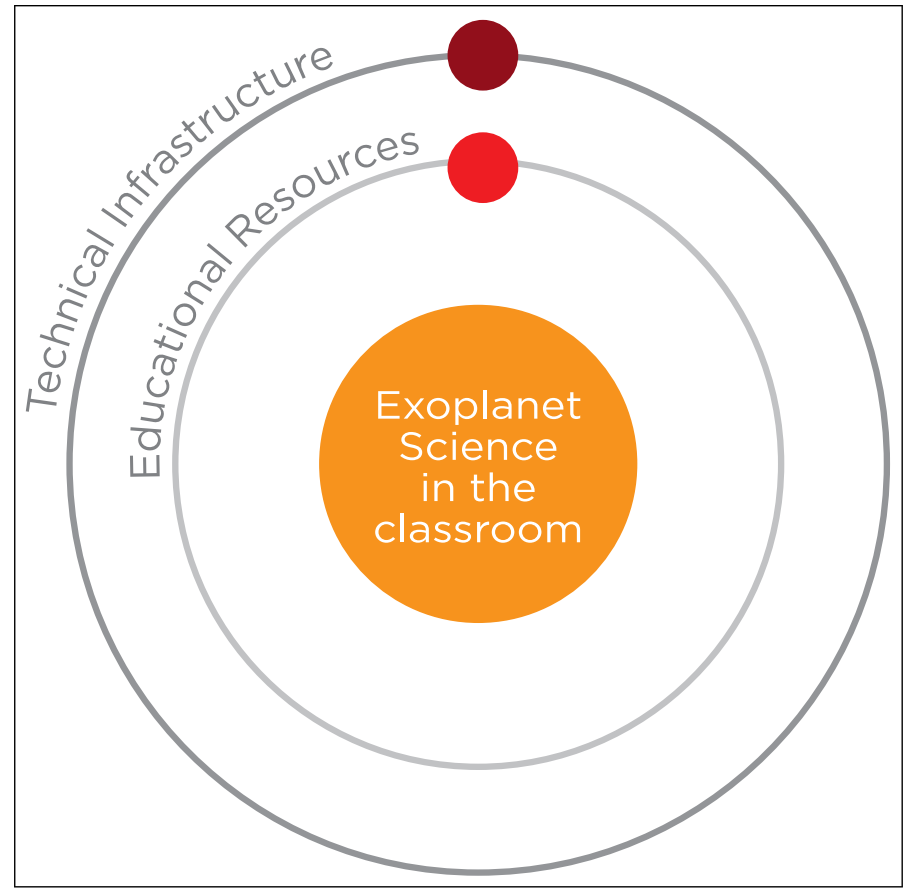

Fig. 6. The educational design for bringing Exoplanet Science into the classroom, is composed of two layers: Technical Infrastructure and Education Resources. conduct viable observations of known exoplanets was compiled. This list is essentially the best targets that can be observed with $0.4 \mathrm{~m}$-class ground-based telescopes. The targets were limited to those that were at most 14th magnitude, and had a transit depth of $>1 \%$. Using these parameters the list was narrowed to 68 targets. As the infrastructure available to the authors was based on using the LCO robotic telescope network - containing both Northern and Southern observatories, the initial list was not limited by geographical location with respect to the observing limits due to Declination and Latitude. However, for general purposes, that list of targets is provided in Tables 1, 2, 3 in this paper divided into Northern, Equatorial and Southern objects. Figures 8 - 10 provide various descriptive distributions for the final target list of exoplanets.

The process of picking such targets was described in more detail in earlier work by Sarva et al. (2020). That paper provides a step-by-step explanation of the process of picking exoplanets for observing using the NASA Exoplanet Archive. In the current paper, the process has been further simplified by providing a simple list to allow complete beginners and teachers with minimal time to be able to quickly enable students to carry out observations. While this list prioritizes those targets that are easiest to observe for beginning users, it does not prioritise the list by what is most useful to observe for science reasons. This is a continually moving target. For the latest lists of transiting planets that are useful for upcoming space telescope missions, it is best to refer to the project websites of Exoplanet Watch and ExoClock for lists of needed observations. 


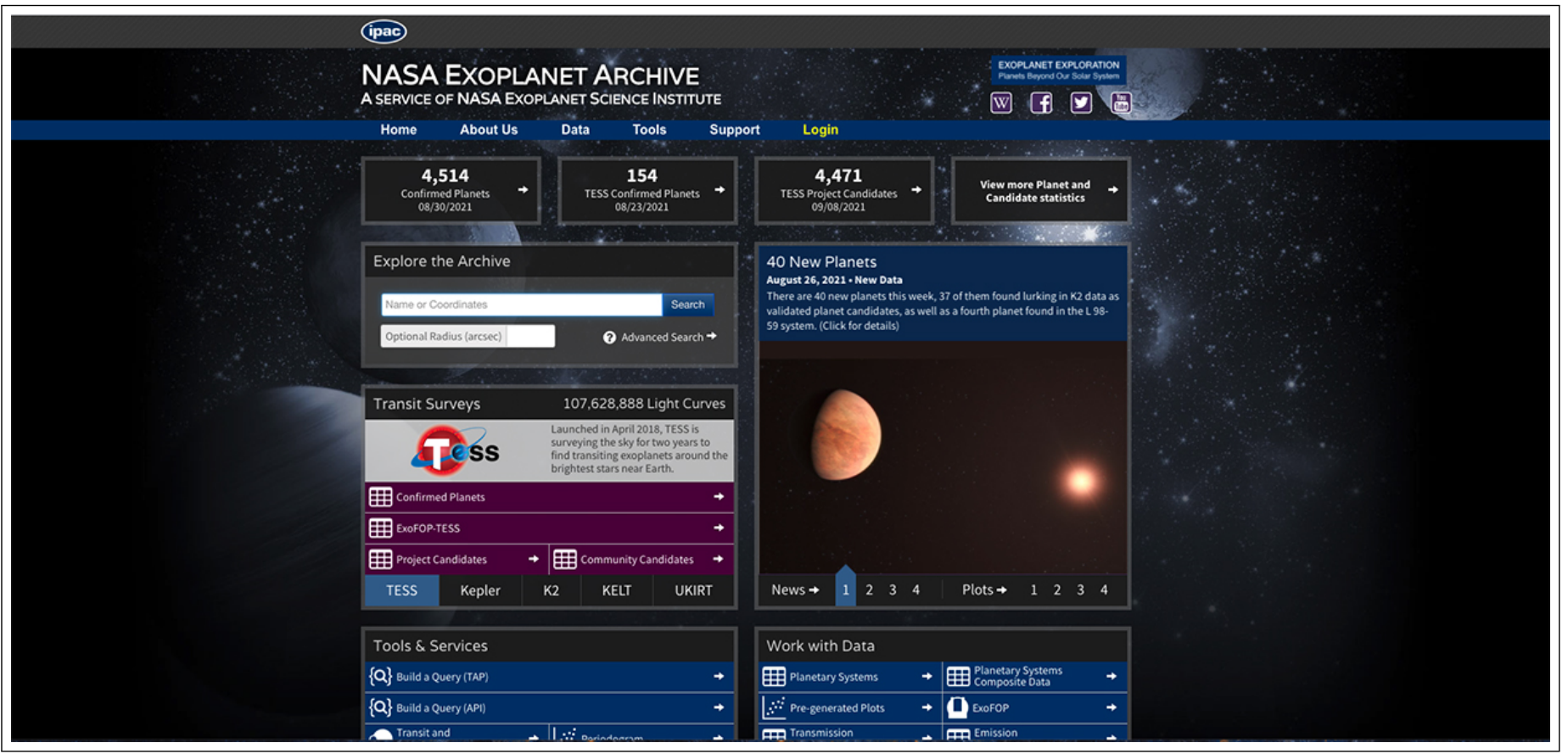

Fig. 7. NASA Exoplanet Archive, a web-based portal that allows access to the most up to date exoplanet catalogue from various missions managed by the California Institute of Technology.

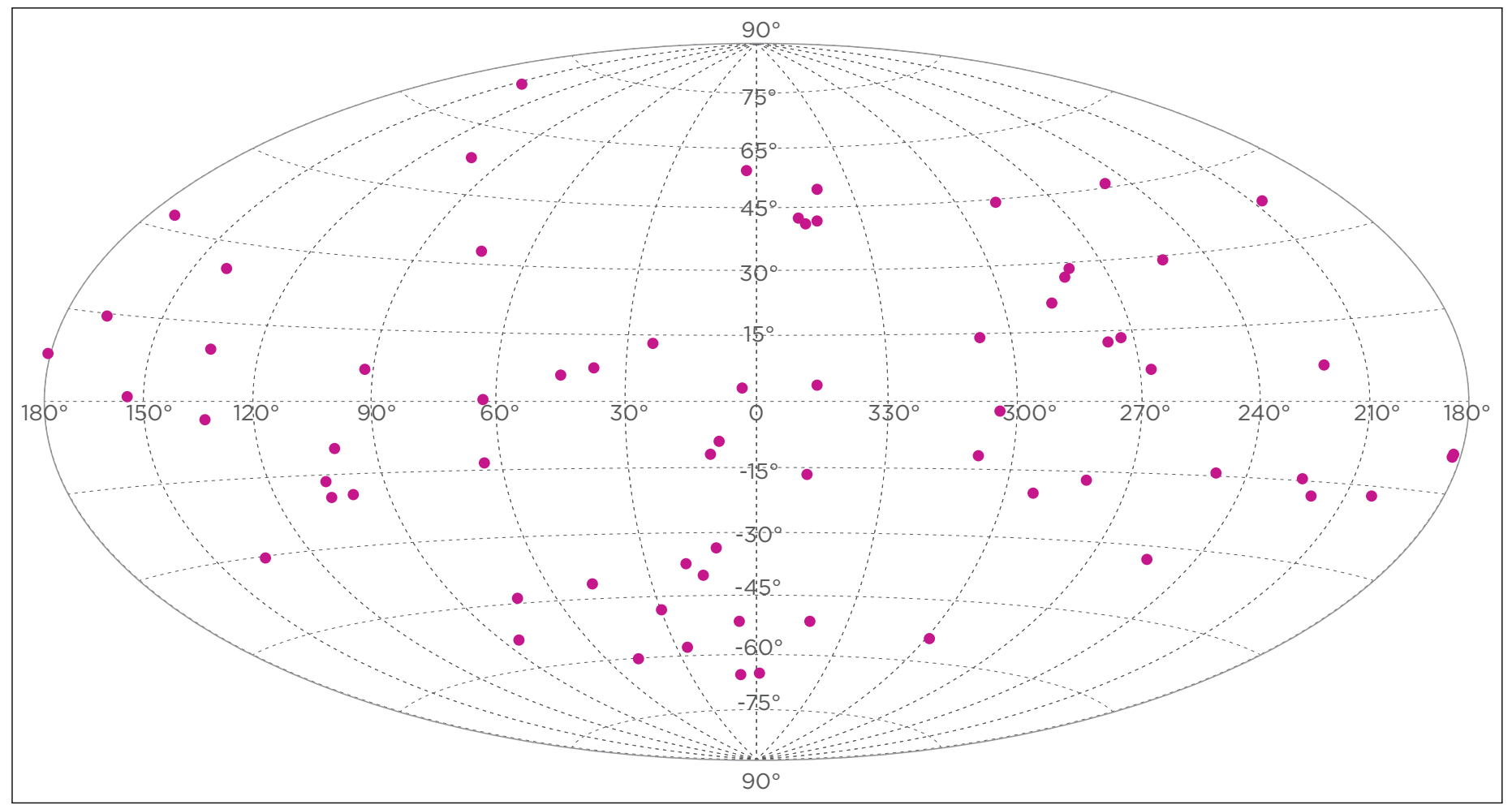

Fig. 8. Distribution of the exoplanets identified in this study in the sky using Aitoff projection with ICRS coordinates in degrees. 


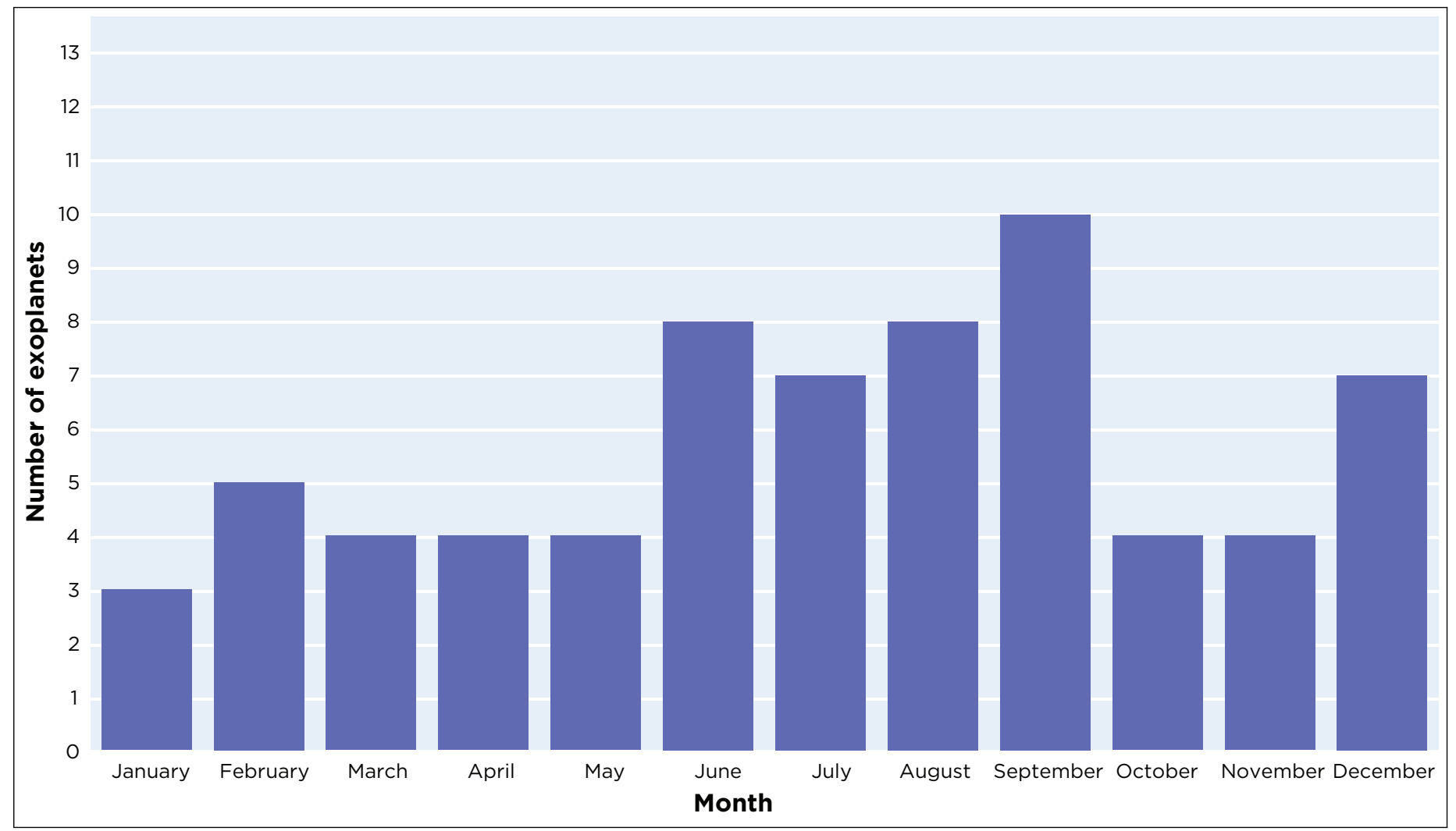

Fig. 9. Distribution of exoplanets that are best observed in particular months derived from their Right Ascension coordinates.

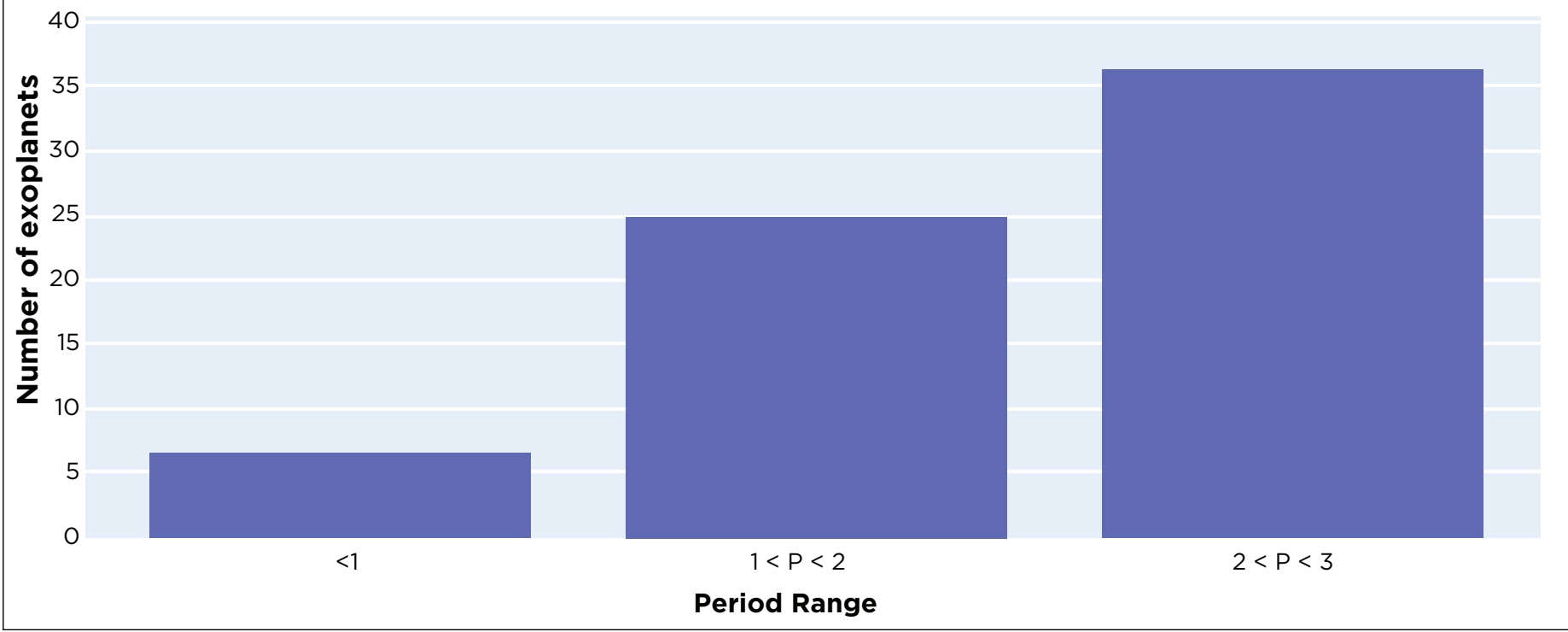

Fig. 10. Distribution of exoplanets for three orbital period bins $<1,1<\mathrm{P}<2$, and $2<\mathrm{P}<3$. The limit to 3 days was to enable students to easily obtain an exoplanet transit within the time-constraints of a semester. 
Table 1. List of exoplanet targets binned into Southern declination zone for declinations less than $-20^{\circ}$.

\begin{tabular}{|c|c|c|c|c|c|c|c|}
\hline \multicolumn{8}{|c|}{ Southern $\left(\right.$ Dec $\left.<-20^{\circ}\right)$} \\
\hline $\begin{array}{l}\text { Planet } \\
\text { Name }\end{array}$ & $\begin{array}{c}\text { RA } \\
\text { (deg) }\end{array}$ & $\begin{array}{l}\text { DEC } \\
\text { (deg) }\end{array}$ & $\begin{array}{c}\text { Best } \\
\text { Month }\end{array}$ & $\begin{array}{l}\text { Orbital } \\
\text { Period } \\
\text { (days) }\end{array}$ & $\begin{array}{c}\text { Transit } \\
\text { duration } \\
\text { (hrs) }\end{array}$ & $\begin{array}{c}\text { Transit } \\
\text { Midpoint } \\
\text { (BJD) }\end{array}$ & $\begin{array}{l}\text { Magnitude } \\
\text { (mag) }\end{array}$ \\
\hline HIP 65 A b & 0.1856063 & -54.830823 & Sep & 0.9809734 & 0.78576 & 2458326.10418 & 11 \\
\hline WASP-97 b & 24.6051389 & -55.772001 & Sep & 2.07276 & 2.5824 & 2456438.18683 & 10.57 \\
\hline WASP-119 b & 55.9336392 & -65.19378 & Oct & 2.4998048 & 2.88 & 2456537.54930 & 12.314 \\
\hline WASP-98 b & 58.4290113 & -34.328272 & Nov & 2.96264 & 1.908 & 2456333.39130 & 13.252 \\
\hline WASP-140 b & 60.3855387 & -20.450999 & Nov & 2.2359835 & 1.5144 & 2456912.35105 & 11.118 \\
\hline TOI-157 b & 73.7014398 & -76.680606 & Nov & 2.0845435 & 2.14584 & 2458326.54771 & 12.725 \\
\hline WASP-64 b & 101.114922 & -32.858388 & Dec & 1.5732918 & 2.39976 & 2455582.60169 & 12.704 \\
\hline WASP-23 b & 101.127503 & -42.76223 & Dec & 2.9444256 & 2.39424 & 2455320.12363 & 12.539 \\
\hline WASP-121 b & 107.600231 & -39.097271 & Dec & 1.2749255 & 2.8872 & 2456635.70832 & 10.514 \\
\hline KELT-14 b & 108.301409 & -42.409762 & Dec & 1.7100588 & 2.1336 & 2457091.02863 & 11.001 \\
\hline WASP-170 b & 135.416303 & -20.720369 & Jan & 2.34478022 & 2.04 & 2457802.39150 & 12.613 \\
\hline WASP-19 b & 148.416767 & -45.659108 & Feb & 0.78884 & 1.572 & 2455168.96801 & 12.248 \\
\hline WASP-123 b & 289.479212 & -32.860124 & Jun & 2.9776412 & 3.0936 & 2456845.17082 & 11.03 \\
\hline WASP-46 b & 318.737012 & -55.871863 & Jul & 1.43037 & 1.67352 & 2455392.31553 & 13.043 \\
\hline WASP-144 b & 320.762876 & -40.048439 & Jul & 2.2783152 & 1.9536 & 2457157.27493 & 13.085 \\
\hline WASP-145 A b & 322.253764 & -58.836147 & Jul & 1.7690381 & 0.9768 & 2456844.16526 & 11.636 \\
\hline WASP-95 b & 337.457824 & -48.003099 & Aug & 2.184673 & 2.784 & 2456338.45851 & 10.092 \\
\hline WASP-164 b & 344.873588 & -60.447819 & Aug & 1.7771255 & 1.60368 & 2457203.85378 & 12.603 \\
\hline WASP-4 b & 353.562915 & -42.061779 & Aug & 1.3382299 & 2.12832 & 2454387.32779 & 12.483 \\
\hline WASP-173 A b & 354.168696 & -34.611304 & Aug & 1.3866529 & 2.3544 & 2458105.59824 & 11.15 \\
\hline WASP-91 b & 357.846434 & -70.152862 & Aug & 2.798581 & 2.3424 & 2456297.71900 & 11.98 \\
\hline WASP-5 b & 359.349028 & -41.27722 & Aug & 1.6284229 & 2.3712 & 2454373.99598 & 12.147 \\
\hline
\end{tabular}


Table 2. List of exoplanet targets binned into Equatorial declination Zone for declinations less between $-20^{\circ}$ and $+20^{\circ}$

Equatorial $(-20<$ Dec $<+20)$

\begin{tabular}{|c|c|c|c|c|c|c|c|}
\hline $\begin{array}{l}\text { Planet } \\
\text { Name }\end{array}$ & $\begin{array}{c}\text { RA } \\
\text { (deg) }\end{array}$ & $\begin{array}{l}\text { DEC } \\
\text { (deg) }\end{array}$ & $\begin{array}{c}\text { Best } \\
\text { Month }\end{array}$ & $\begin{array}{l}\text { Orbital } \\
\text { Period } \\
\text { (days) }\end{array}$ & $\begin{array}{c}\text { Transit } \\
\text { duration } \\
\text { (hrs) }\end{array}$ & $\begin{array}{c}\text { Transit } \\
\text { Midpoint } \\
\text { (BJD) }\end{array}$ & $\begin{array}{c}\text { Magnitude } \\
\text { (mag) }\end{array}$ \\
\hline WASP-44 b & 3.9032733 & -11.938265 & Sep & 2.423804 & 2.2368 & 2455434.37600 & 13.096 \\
\hline WASP-32 b & 3.9617073 & 1.2005122 & Sep & 2.718659 & 2.424 & 2455151.05460 & 11.257 \\
\hline WASP-26 b & 4.6030424 & -15.267404 & Sep & 2.7566004 & 2.3832 & 2455228.38842 & 11.297 \\
\hline WASP-76 b & 26.632936 & 2.700389 & Sep & 1.809886 & 3.6936 & 2456107.85507 & 9.518 \\
\hline WASP-77 A b & 37.1555223 & -7.0606675 & Oct & 1.3600309 & 2.16 & 2455870.44977 & 10.294 \\
\hline WASP-50 b & 43.6880738 & -10.898024 & Oct & 1.9550959 & 1.80576 & 2455558.61197 & 11.44 \\
\hline HAT-P-70 b & 74.5523332 & 9.9979794 & Nov & 2.744321 & 3.421 & 2459197.00754 & 9.47 \\
\hline WASP-49 b & 91.0897193 & -16.96539 & Dec & 2.7817362 & 2.14 & 2455580.59436 & 11.352 \\
\hline WASP-36 b & 131.58039 & -8.0269855 & Jan & 1.5373653 & 1.81584 & 2455569.83731 & 12.836 \\
\hline WASP-65 b & 133.324298 & 8.5230171 & Jan & 2.3114243 & 2.73504 & 2456110.68772 & 11.869 \\
\hline WASP-43 b & 154.908187 & -9.8064431 & Feb & 0.813475 & 1.1592 & 2455528.86774 & 12.305 \\
\hline WASP-104 b & 160.602369 & 7.4350768 & Feb & 1.7554137 & 1.76208 & 2456406.11126 & 11.779 \\
\hline WASP-85 A b & 175.908033 & 6.5637842 & Feb & 2.6556777 & 2.59584 & 2456847.47286 & 10.72 \\
\hline $\mathrm{K} 2-229 \mathrm{~b}$ & 186.87292 & -6.7218474 & Mar & 0.58426 & 1.5 & 2457583.46910 & 10.985 \\
\hline $\mathrm{K} 2-228 \mathrm{~b}$ & 187.294705 & -6.8342028 & Mar & 2.69828 & 1.5 & 2457583.19430 & 13.028 \\
\hline Qatar-2 b & 207.655493 & -6.8040714 & Mar & 1.33711677 & 1.809816 & 2457250.20082 & 13.443 \\
\hline WASP-57 b & 223.819979 & -2.0576866 & Apr & 2.838971 & 2.304 & 2455717.87811 & 12.913 \\
\hline WASP-24 b & 227.215498 & 2.343286 & Apr & 2.34121242 & 2.6832 & 2455081.37941 & 11.219 \\
\hline WASP-103 b & 249.31486 & 7.1833758 & May & 0.925542 & 2.593 & 2456459.59957 & 12.402 \\
\hline WASP-163 b & 256.537555 & -10.413007 & May & 1.6096884 & 2.232 & 2457918.46200 & 12.663 \\
\hline CoRoT-11 b & 280.6873 & 5.9376586 & Jun & 2.99433 & 2.5009 & 2454597.67900 & 12.897 \\
\hline CoRoT-2 b & 291.777046 & 1.3836634 & Jun & 1.7429935 & 2.26704 & 2454237.53562 & 12.516 \\
\hline HAT-P-23 b & 306.123908 & 16.7621462 & Jul & 1.212884 & 2.1792 & 2454852.26464 & 11.937 \\
\hline WASP-2 b & 307.725559 & 6.4293305 & Jul & 2.152175 & 1.78824 & 2458339.00342 & 11.728 \\
\hline WASP-75 b & 342.3859 & -10.675469 & Aug & 2.484193 & 1.9728 & 2456016.26690 & 11.591 \\
\hline WASP-52 b & 348.494793 & 8.7610793 & Aug & 1.7497798 & 1.8096 & 2455793.68143 & 12.192 \\
\hline
\end{tabular}


Table 3. List of exoplanet targets binned into Northern declination zone for declinations greater than $+20^{\circ}$.

\begin{tabular}{|c|c|c|c|c|c|c|c|}
\hline \multicolumn{8}{|c|}{ Northern $\left(\right.$ Dec $\left.>+20^{\circ}\right)$} \\
\hline $\begin{array}{l}\text { Planet } \\
\text { Name }\end{array}$ & $\begin{array}{c}\text { RA } \\
\text { (deg) }\end{array}$ & $\begin{array}{l}\text { DEC } \\
\text { (deg) }\end{array}$ & $\begin{array}{c}\text { Best } \\
\text { Month }\end{array}$ & $\begin{array}{l}\text { Orbital } \\
\text { Period } \\
\text { (days) }\end{array}$ & $\begin{array}{c}\text { Transit } \\
\text { duration } \\
\text { (hrs) }\end{array}$ & $\begin{array}{c}\text { Transit } \\
\text { Midpoint } \\
\text { (BJD) }\end{array}$ & $\begin{array}{l}\text { Magnitude } \\
\text { (mag) }\end{array}$ \\
\hline Qatar-4 b & 4.859275 & 44.0275965 & Sep & 1.8053564 & 2.1384 & 2457637.77361 & 13.574 \\
\hline Qatar-5 b & 7.0539375 & 42.0613451 & Sep & 2.8792319 & 2.9088 & 2457336.75824 & 12.614 \\
\hline WASP-93 b & 9.4587443 & 51.288778 & Sep & 2.7325321 & 2.2344 & 2456079.56420 & 10.966 \\
\hline HAT-P-16 b & 9.5730343 & 42.4630961 & Sep & 2.77596 & 3.0624 & 2455027.59293 & 10.911 \\
\hline HAT-P-32 b & 31.0427614 & 46.6878512 & Oct & 2.1500082 & 3.12048 & 2455867.40274 & 11.439 \\
\hline WASP-12 b & 97.636645 & 29.6722662 & Dec & 1.0914203 & 2.99592 & 2456176.66826 & 11.569 \\
\hline $\mathrm{XO}-2 \mathrm{Nb}$ & 117.026769 & 50.2251472 & Dec & 2.615826 & 2.653041 & 2458843.21868 & 11.246 \\
\hline KELT-4 A b & 157.06262 & 25.5731366 & Feb & 2.9895932 & 3.46272 & 2456190.30201 & 9.98 \\
\hline HAT-P-36 b & 188.266205 & 44.9153672 & Mar & 1.3273466 & 2.23248 & 2456698.73591 & 12.146 \\
\hline WASP-14 b & 218.276625 & 21.8946875 & Apr & 2.243752 & 3.06 & 2454463.57583 & 9.745 \\
\hline KELT-23 A b & 232.146641 & 66.3587097 & Apr & 2.25528783 & 2.278 & 2458140.38698 & 10.308 \\
\hline WASP-92 b & 246.69204 & 51.0411328 & May & 2.1746742 & 2.7672 & 2456381.28340 & 12.951 \\
\hline WASP-135 b & 267.284885 & 29.8790428 & May & 1.4013794 & 1.656 & 2455230.99020 & 13.181 \\
\hline HAT-P-5 b & 274.405534 & 36.6214617 & Jun & 2.788491 & 2.9208 & 2454241.77663 & 11.954 \\
\hline WASP-3 b & 278.631741 & 35.6614312 & Jun & 1.8468355 & 2.772 & 2454640.64993 & 10.632 \\
\hline HAT-P-37 b & 284.296039 & 51.2691212 & Jun & 2.797436 & 2.3304 & 2455642.14000 & 13.427 \\
\hline TrES-2 b & 286.808526 & 49.3164211 & Jun & 2.47061892 & 1.789 & 2455642.14318 & 11.254 \\
\hline Kepler-854 b & 293.351268 & 43.1346404 & Jun & 2.14463285 & 3.9028 & 2454966.98434 & 13.417 \\
\hline Qatar-1 b & 303.38187 & 65.1623313 & Jul & 1.4200242 & 1.66104 & 2456234.10322 & 12.692 \\
\hline KELT-16 b & 314.268523 & 31.6610186 & Jul & 0.9689951 & 2.4888 & 2457247.24791 & 11.717 \\
\hline
\end{tabular}




\section{User Interface}

Another challenging aspect of making exoplanet observations is being able to observe a complete transit. This requires knowing the location of the host star on the celestial sphere and the orbital period and the Transit mid-point of the exoplanet, among other parameters. Next, it requires carefully planning the observation such that a complete transit can be observed within the available time frame. This can be challenging for students and teachers, as this requires very deep conceptual and content knowledge as well as applying an algorithm that can confuse experts at times!

\section{MANUALLY CALCULATING A FUTURE TRAN- SIT TIME}

There are a variety of online transit calculators, such as Tapir at Swarthmore (Jensen, 2013), that will provide available transits to observe at your location according to specified limits. In the future are all possible observable transits that we would like to predict. In the past some of the previous transits have been observed, allowing us to estimate how often the transit occurs (orbital period) and when (transit midpoint). From Figure 11, if we know any previous time that the transit occurred (the "midpoint transit time"), we can simply keep adding the orbital period onto this time until the transit occurs in the future from now.

Once we know that we have a transit that is in the future (and we have checked that it is occuring at our nighttime... otherwise we move on and check the next transit!) then, knowing the transit duration, we can estimate appropriate start and stop times for a transit observation, which are usually equally distant in time from the midpoint transit time. This is shown schematically in Figure 12 from Sarva et al. (2020). Having figured out all of this timing, the last important piece of information is the star's brightness from its optical magnitude, which allow us to estimate a long enough, but not too long, exposure time suitable for our target exoplanet star. Assuming that we have selected a star that has a deep enough transit to be observed (all stars in the provided target list in this paper are $>1 \%$ ), then from all this information we can program in our observation to a robotic telescope or prepare to manually observe the transit.

The observation start time is the predicted midpoint transit time minus half the transit duration, minus the amount of time to observe outside the transit event when the transit curve is "flat", minus a small amount of buffer time to help with scheduling or control for shifts in the midpoint transit. The observation end time is the point equally distant in time from the midpoint transit time, but after the transit. On the face of it, it may not seem too confusing but there are quite a number of calculation steps that can be prone to calculation error for beginners and experts alike.

\section{ExoRequest}

To simplify and automate these steps, an automated observation routine has been developed - ExoRequest - written in Python, which can be implemented via Google Colaboratory (see Figure 13). ExoRequest can also be run locally on Windows or macOS. It can be used to submit directly to Las Cumbres Observatory (LCO) or be used for planning for a local observatory. ExoRequest requires that the user knows the necessary parameters (such as those in the provided tables) for their given exoplanet. As well as the typical parameters, such as midtransit point and period, it also estimates a reasonable exposure time based on the provided optical magnitude. This information is used to calculate the relevant details described above and automatically submit this to the LCO scheduler.

\section{ExoSelect: Web-based observation request portal}

When the user does not know what object they might like to observe, or wants to select from some wellknown targets for that time of the year, a further Web-based interface - ExoSelect - has been created to automatically fill in these values in (see Figure 14). ExoSelect also functions as a web interface to ExoRequest by using a manual drop-down box. This brings in an additional layer of simplicity, especially for instances where students may not have access to the technology infrastructure, or the confidence to run computer code. The web-based interface was developed using VueJS, wrapped in an HTML5 page, and Flask/Python which allows the observation portal to pull information from the web-based user interface and send it to the LCO observing portal. ExoSelect sends the required information to ExoRequest in Python using Flask, which then sends the information to the LCO observing portal (see Figure 15). In addition, there is a semi-automated pipeline that allows the maintainers of the website to dynamically send an updated list of exoplanets to ExoSelect, ensuring that new potential exoplanets are made available to students. 


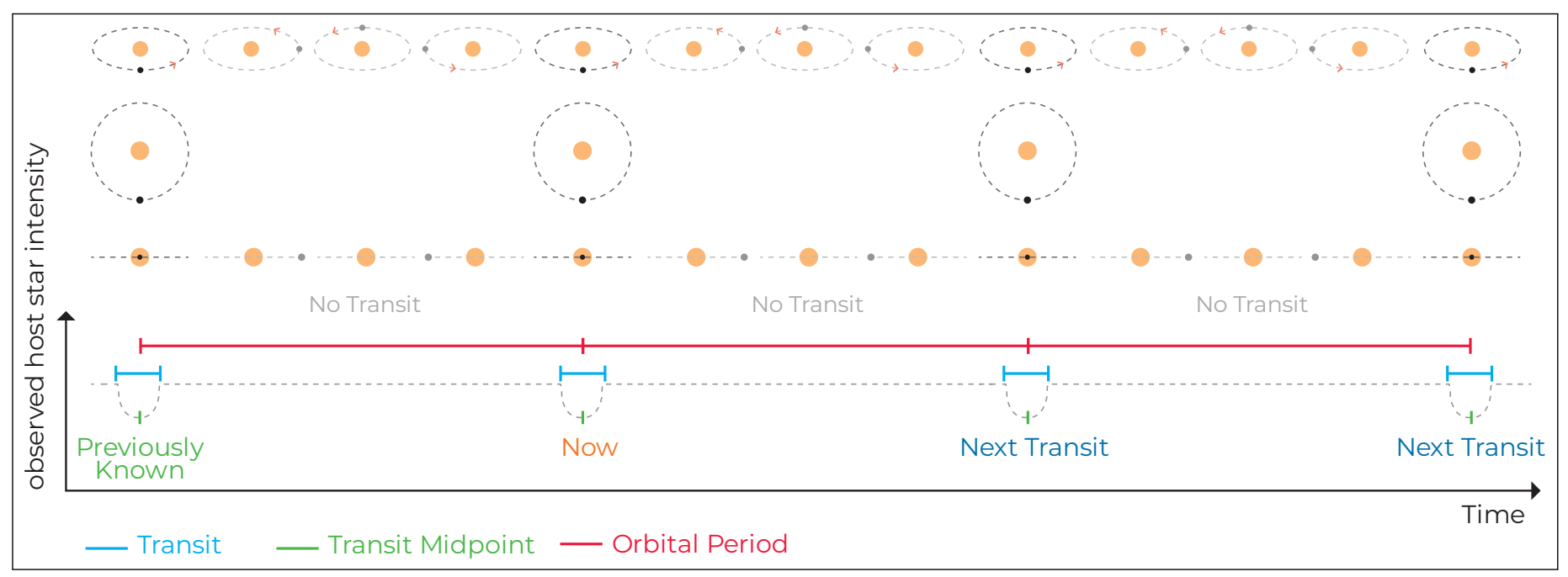

Fig. 11. A simplified schematic showing the fundamental method behind automatically calculating upcoming transmits. The red bars represent the period of the orbit. The blue bars represent the transit duration. Knowing the Transit midpoint, orbital period and transit duration retrieved from the NASA Exoplanet Archive, every upcoming transit from the current date can be calculated. Figure not to scale.

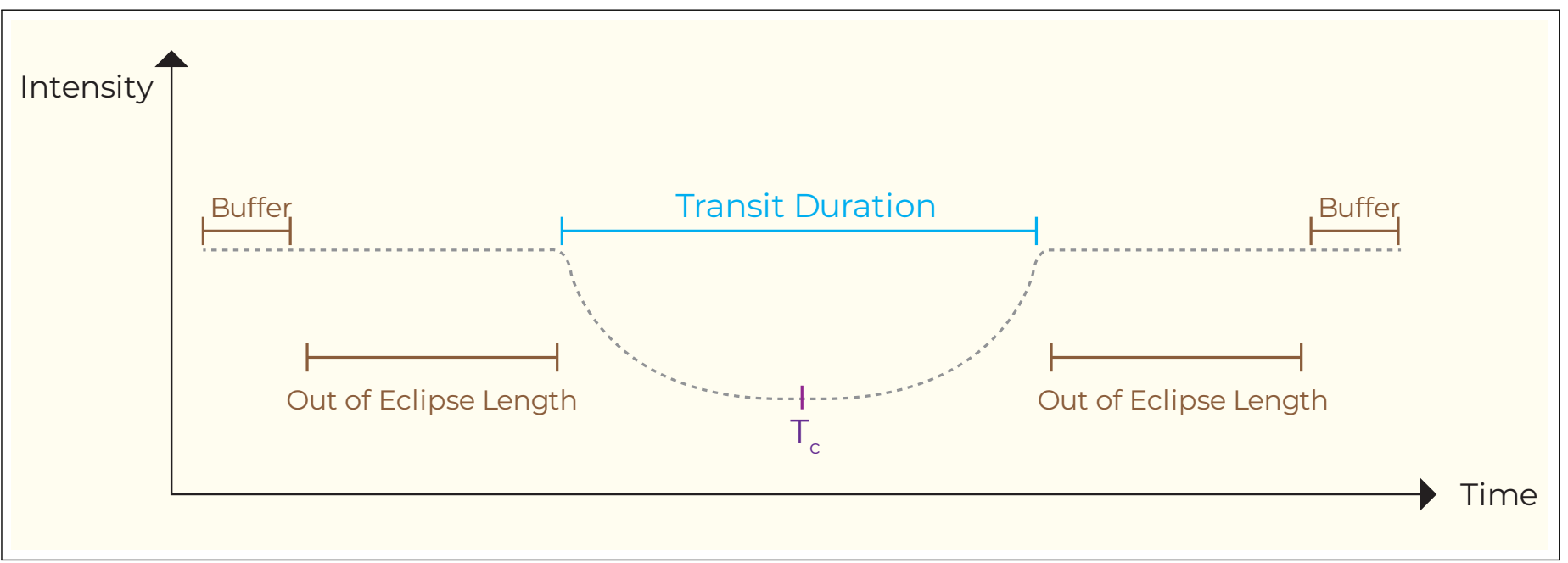

Fig. 12. A schematic illustrating the considerations necessary to determine the start and stop times for an exoplanet transit observation from Sarva et al. (2020). 


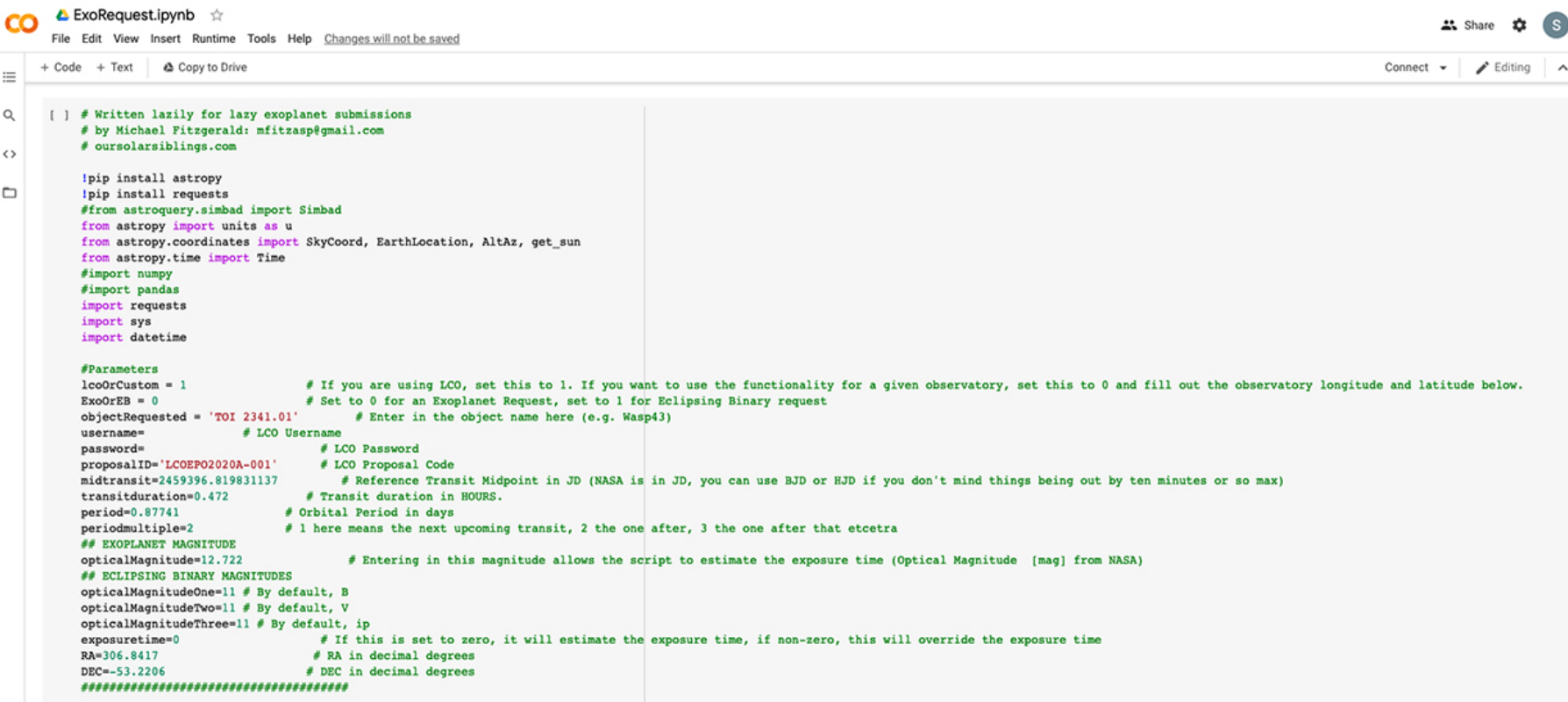

Fig. 13. ExoRequest in Google Collaboratory. ExoRequest, a script written in Python, automatically schedules observations of exoplanets. taking away the time consuming challenge of planning observations to be able to observe a complete transit.

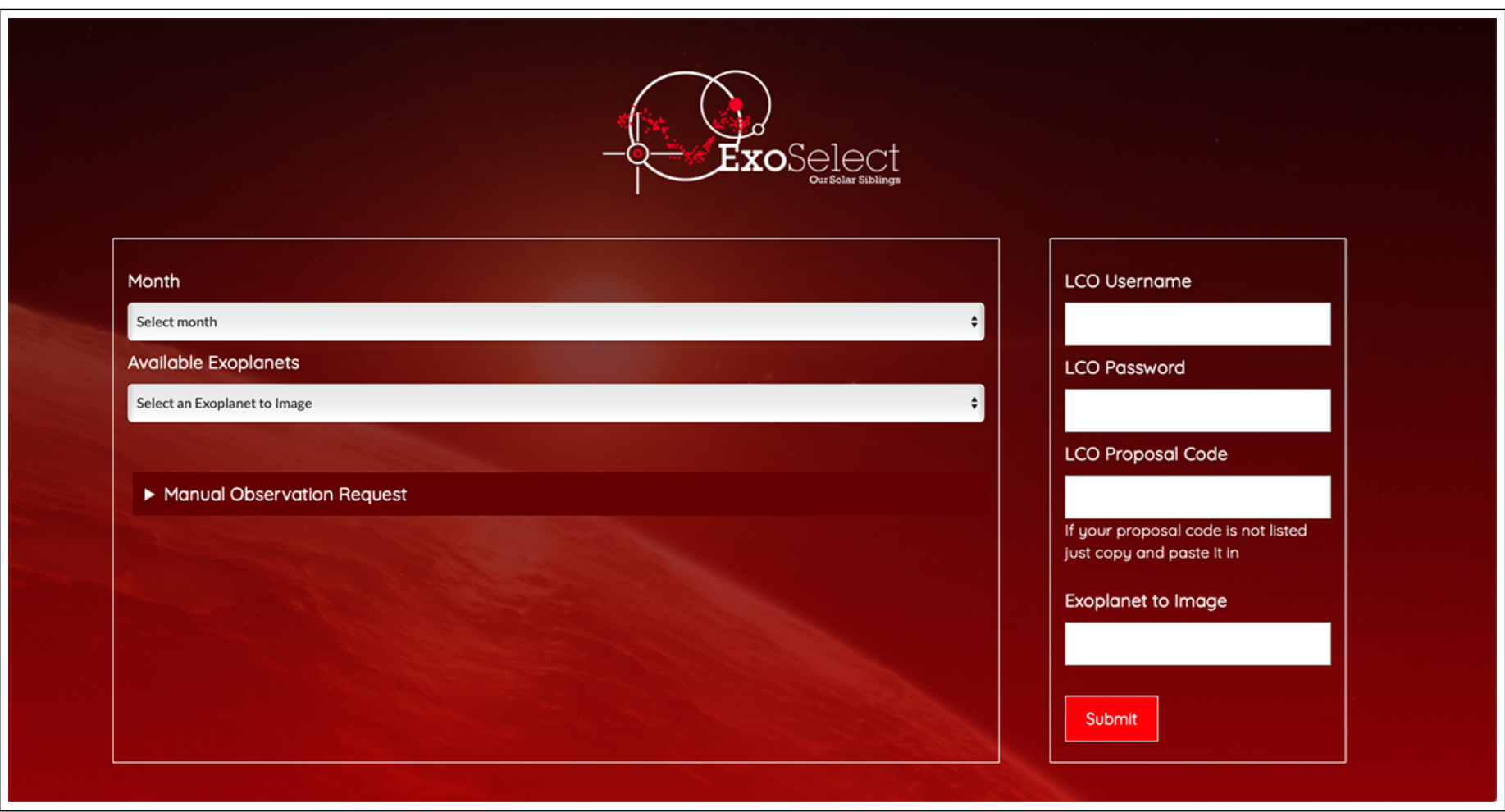

Fig. 14. ExoSelect, web-based portal for requesting observations of exoplanets. http://exoselect.herokuapp.com 


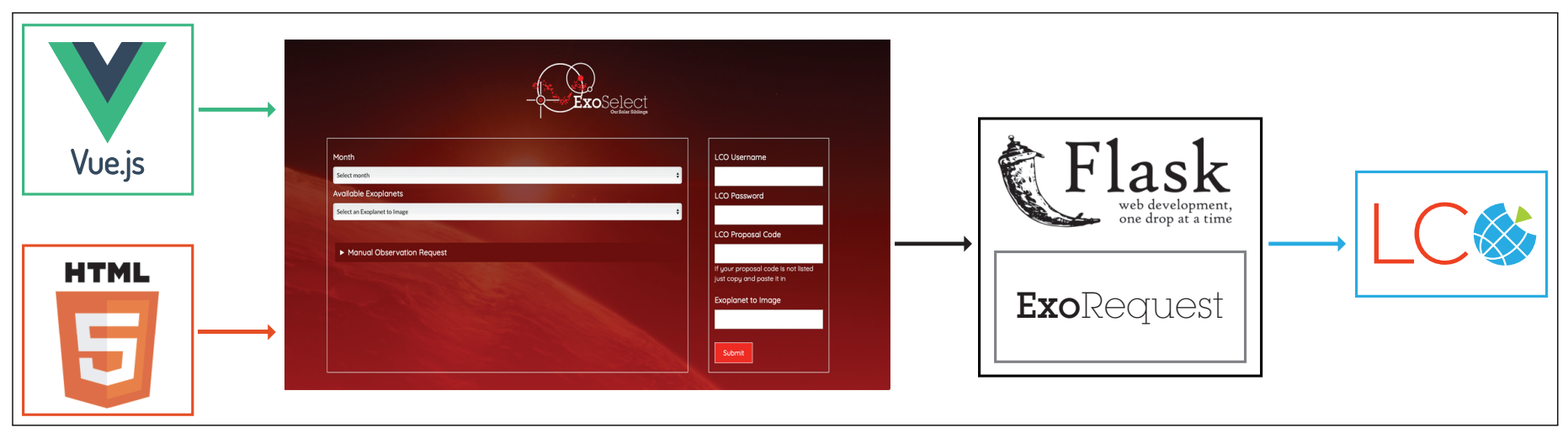

Fig. 15. The technical infrastructure for quickly picking exoplanets and scheduling observations of exoplanets using the LCO robotic telescope network.

\section{EDUCATIONAL CONSIDERATIONS}

Thinking through what can be done in a given educational context involves a cost/benefit analysis. One of the significant costs is the amount of conceptual development and mathematical skill required on the part of the user to predict and plan a future exoplanet observation. The actual complexity of exploring and analysing the transit observation data itself is comparatively straightforward, especially with new software tools such as EXOTIC (Zellem et al., 2020) and HOPS (Tsiaras, 2019) becoming available. Without tools like these, and planning tools such as ExoRequest and ExoSelect, contributions by keen (but time-poor) students can really only be done at the Citizen Science level or with pre-observed data near the top of the Rebull funnel (Rebull, 2018). With the provided tools, however, students are enabled to collect and analyse data the "counts" by contributing to actual science through providing important data necessary to plan space telescope observation time.

By providing the observing planning tools, this facilitates the capacity of students to contribute real meaningful data, at the most basic level of transit timing (e.g. Agol \& Fabrycky, 2018; Baştürk et al., 2019; Steffen et al., 2007). When combined with other tools, this can provide capacity to delve even deeper into some original research. For some educators, the planning of the observation is an important part of the process contributing to building scientific planning skills for the student. For others this is a limiting factor in terms of time and too steep a learning curve for their students, who may want a more simple exploration of an exoplanet transit. By providing such tools that automate the planning aspect, students and users towards the top of the Rebull funnel are supported in their explorations.

\section{CONCLUSION}

The aim of this paper was to introduce the technical infrastructure (ExoRequest and ExoSelect) that has been developed to streamline requesting observations of exoplanets for use in the classroom environment where time is of the essence. This is further facilitated by provision of a select list of best targets, ordered by month of the year accessible in tables in this paper, as well as through the online ExoSelect interface. In providing such support, students can focus on understanding the conceptual aspects of exoplanet science and analysis, while still engaging in authentic inquiry.

\section{FUNDING}

No funding was received for this project.

\section{ACKNOWLEDGMENTS}

Thanks to the teachers and students who have trialled the many iterations of ExoSelect.

\section{REFERENCES}

Agol, E., \& Fabrycky, D. C. (2018). Transit-timing and duration variations for the discovery and characterization of exoplanets. Handbook of Exoplanets, 7.

Baştürk, Ö., Esmer, E. M., Torun, Ş., Yalçınkaya, S., Helweh, F. E., Karamanl, E., ... others (2019). Transit timing variations of five transiting planets. In Aip conference proceedings (Vol. 2178, p. 030019). 
Borucki, W. J., Koch, D., Basri, G., Batalha, N., Brown, T., Caldwell, D., ... others (2010). Kepler planetdetection mission: introduction and first results. Science, 327(5968), 977-980.

Brown, T., Baliber, N., Bianco, F., Bowman, M., Burleson, B., Conway, P., ... others (2013). Las cumbres observatory global telescope network. Publications of the Astronomical Society of the Pacific, 125(931), 1031.

Fitzgerald, M. T. (2018). The our solar siblings pipeline: Tackling the data issues of the scaling problem for robotic telescope based astronomy education projects. RTSRE Proceedings, 1(1).

Fitzgerald, M. T., Danaia, L., \& McKinnon, D. H. (2019). Barriers inhibiting inquiry-based science teaching and potential solutions: perceptions of positively inclined early adopters. Research in Science Education, 49(2), 543-566.

Fitzgerald, M. T., Hollow, R., Rebull, L. M., Danaia, L., \& McKinnon, D. H. (2014). A review of high school level astronomy student research projects over the last two decades. Publications of the Astronomical Society of Australia, 31.

Fitzgerald, M. T., McKinnon, D. H., \& Danaia, L. (2015). Inquiry-based educational design for largescale high school astronomy projects using real telescopes. Journal of Science Education and Technology, 24(6), 747-760.

Fitzgerald, M. T., McKinnon, D. H., Danaia, L., Cutts, K. R., Salimpour, S., \& Sacchi, M. (2018). Our solar siblings: A high school focused robotic telescopebased astronomy education project. RTSRE Proceedings, 1(1).

Gomez, E. L., \& Fitzgerald, M. T. (2017). Robotic telescopes in education. Astronomical Review, 13(1), $28-68$.

Guerrero, N. M., Seager, S., Huang, C. X., Vanderburg, A., Soto, A. G., Mireles, I., ... others (2021). The tess objects of interest catalog from the tess prime mission. The Astrophysical Journal Supplement Series, 254(2), 39.

Howell, S. B. (Ed.). (2020). The nasa kepler mission. IOP Publishing. Retrieved from http://dx.doi . org/10.1088/2514-3433/ab9823 doi:
Jensen, E. (2013). Tapir: A web interface for transit/eclipse observability. Astrophysics Source Code Library, ascl-1306.

Lehrer, R., \& Schauble, L. (2007). Scientific thinking and science literacy. Handbook of Child Psychology, 4.

Luckas, P., \& Gottschalk, K. (2018). The spirit telescope initiative: Engaging students in contemporary astronomy. RTSRE Proceedings, 1(1).

Luft, J. A., Hanuscin, D., Hobbs, L., \& Törner, G. (2020). Out-of-field teaching in science: An overlooked problem. Taylor \& Francis.

Rebull, L. (2018). Authentic research in the classroom for teachers and students. Robotic Telescope, Student Research and Education Proceedings, 1(1), 21-31.

Reichart, D., Nysewander, M., Moran, J., Bartelme, J., Bayliss, M., Foster, A., ... others (2005). Prompt: panchromatic robotic optical monitoring and polarimetry telescopes. arXiv preprint astroph/0502429.

Ricker, G. R., Latham, D., Vanderspek, R., Ennico, K., Bakos, G., Brown, T., ... others (2010). Transiting exoplanet survey satellite (tess). In American astronomical society meeting abstracts\# 215 (Vol. 215, pp. 450-06).

Roche, P., Roberts, S. E., Gomez, E. L., Tripp, A., Lewis, F., Stroud, V., ... Tryfona, C. (2008). Education and public outreach programmes for the faulkes telescope project. In R. J. Simpson \& D. Ward-Thompson (Eds.), Astronomy: networked astronomy and the new media. Canopus publishing.

Sadler, P. M., Gould, R. R., Leiker, P. S., Antonucci, P. R., Kimberk, R., Deutsch, F. S., ... others (2001). Microobservatory net: A network of automated remote telescopes dedicated to educational use. Journal of Science Education and Technology, 10(1), 39-55.

Salimpour, S., Bartlett, S., Fitzgerald, M. T., McKinnon, D. H., Cutts, K. R., James, C. R., ... others (2020). The gateway science: A review of astronomy in the oecd school curricula, including china and south africa. Research in Science Education, $1-22$. 
Salimpour, S., Tytler, R., Eriksson, U., \& Fitzgerald, M. T. (2021). Cosmos visualized: Development of a qualitative framework for analyzing representations in cosmology education. Physical Review Physics Education Research, 17(1), 013104.

Sarva, J., Freed, R., Fitzgerald, M. T., \& Salimpour, S. (2020). An exoplanet transit observing method using lco telescopes, exorequest and astrosource. Astronomy Theory, Observations \& Methods, 1(1).

Shulman, L. S. (1986). Those who understand: Knowledge growth in teaching. Educational researcher, 15(2), 4-14.

Steffen, J. H., Gaudi, B. S., Ford, E. B., Agol, E., \& Holman, M. J. (2007). Detecting and characterizing planetary systems with transit timing. arXiv preprint arXiv:0704.0632.

Tsiaras, A. (2019, September). HOPS: the photometric software of the HOlomon Astronomical Station. In Epsc-dps joint meeting 2019 (Vol. 2019, p. EPSCDPS2019-1594).

Zellem, R. T., Pearson, K. A., Blaser, E., Fowler, M., Ciardi, D. R., Biferno, A., ... others (2020). Utilizing small telescopes operated by citizen scientists for transiting exoplanet follow-up. Publications of the Astronomical Society of the Pacific, 132(1011), 054401. 